\title{
DER EINFLUSS DEUTSCHER STAATSLEHREN \\ AUF DIE NATIONALE INTELLIGENZ IN CHINA IN DER VORREVOLUTIONAREN PHASE \\ NACH 1900
}

Von

RÜDIGER MACHETZKI

Der Jahrhundertprozeß der chinesischen Revolution (1850-1950) hat in vielerlei Hinsicht eine ähnliche Bedeutsamkeit für die asiatische Welt des 20. Jahrhunderts wie die industrielle Revolution in England und die politisch-soziale Revolution in Frankreich für den Westen des 19. Jahrhunderts. Beide Revolutionswellen liefen in der Entstehung neuer, machtvoller Staatsgebilde aus, deren gewaltige Grundenergien sich wirtschaftlich in nationalem „Wohlstand und Macht" (Fu-ch'iang) umsetzen sollten. Politisch spiegelten sie sich in einer neuartigen und intensiven nationalen Identität wider, die in der Gestalt wirkungsvoller, staatlicher Institutionen und Organisationen ihren Ausdruck fand.

In China hatte dieser langzeitige Umwälzungsvorgang vor der Mitte des vorigen Jahrhunderts eingesetzt mit der allmählichen Auflösung der traditionellen, sozialen und politischen Ordnungsinstitutionen des Kaiserreiches. Zwischen 1851 und 1864 kam es zu der ersten größeren Revolutionsbewegung, die auf westlichen Vorstellungen von gesellschaftlicher Gerechtigkeit mitbasierte, der sogenannten T'aiP'ing-Revolution. Den Wirren dieser Revolution fielen nicht weniger als 30 bis 35 Millionen Menschen zum Opfer, und noch fünfzig Jahre später hatten einige Provinzen sich von den Zerstörungen dieser Zeit nicht vollständig erholt.

Der weitere Zerfall des Reiches und seiner traditionellen Wirtschaft war nicht zuletzt durch die ständig machtvolleren Interessen der westlichen Mächte beschleunigt worden. Vor allem im Bewußtsein der politisch bedeutungsvollen Gesellschaftsschichten wurde die technologische und militärische Überlegenheit des Westens - durch eine Reihe von Kriegen schmerzvoll unter Beweis gestellt - zu einer demütigend zentralen Problematik. Um die Jahrhundertwende hatte dieser wirtschaftliche und militärische Drucls eine derart gewaltige Dimension erreicht, $\mathrm{da} ß$ einflußreiche, chinesische Bevölkerungsteile in politischen Parteien und Bewegungen ihrer Überzeugung Ausdruck zu verleihen begannen, daß nur die Abkehr von der eigenen Tradition und die revolutionäre Durchsetzung westlicher Ideen und Verhaltensnormen China vor dem endgültigen Untergang bewahren könnte. Von dieser Situation her setzte dann schubweise die Welle politischer und sozialer Revolutionsbewegungen ein, die mit dem Sturz des Kaiserreichs 1911 begann und mit der Gründung der Volksrepublik China ausmündete. Alle diese einander folgenden Bewegungen hatten ein gleichermaßen gemeinsames Ziel auf ihre Fahnen geschrieben, das sie auf verschiedene Weise zu verwirklichen suchten, die wirtschaftliche Stärkung und politisch-nationale Unabhängigkeit Chinas.

Die Geschichte der Revolutionen des 19. Jahrhunderts und des 20. Jahrhunderts zeigt jedoch, daß entgegen weit verbreiteter Ansicht die beiden Prozesse nationalökonomischer Transformation und politisch-nationaler Integration in entscheidenden Phasen häufig gegeneinander wirken. 
Einige wenige, meist küstennahe Kleingebiete entwickeln sich zu urban-industriellen Zentren und werden von den inneren, vormodernen Landregionen ständig zunehmend isoliert. Die wirtschaftliche Modernisierung bedeutet daher in vielen Fällen eher ein Aufbrechen der historischen Einheit und des vornationalen Gemeinsamkeitsbewußtseins. Statt der erhofften nationalen Integration setzt ein $\mathrm{Ab}-$ lauf gesellschaftlich ökonomischen Zerfalls und kultureller Entfremdung zwischen den vormodernen Landmassen und den als feindselig empfundenen, industriellen "Antikörpern" ein.

In China wurde dieser gesellschaftliche Zerstörungs- und Entfremdungsprozeß zur grundlegenden Antriebskraft der bisher letzten Bewegung der chinesischen Revolution, der kommunistischen Partei unter Mao Tse-tung ${ }^{1}$. Um die Jahrhundertwende jedoch war ein wesentlicher Teil der chinesischen, politischen Intelligenz noch zutiefst überzeugt, die politische und wirtschaftliche Umgestaltung Chinas, die nationale "Wiederauferstehung" (Chung-hsing), einleiten zu können, ohne den langwierigen und destruktiven Weg einer sozialen und politischen Revolution begehen zu müssen.

Zwei Länder vor allem übten dabei eine gewaltige Anziehungskraft auf die chinesische Intelligenz aus, Japan und Deutschland. Beide Länder hatten, so schien es, sich aus nationaler Zersplitterung und Ohnmacht erhoben und zu machtvollen Nationalstaaten entwickelt, ohne zuvor eine selbstzerstörerische und leidensvolle politisch-soziale Revolutionsphase erlebt zu haben. Diese Art der inneren, nationalen „Reinigung" und Festigung unter Führung der traditionellen Herrschaftseliten und Regierungsinstitutionen, kurzum die „konservative“ Modernisierung, erschien einem Großteil der chinesischen Intelligenz um so unausweichlicher, als der imperialistische Druck der Mächte um die Jahrhundertwende einen für China qualvollen Höhepunkt erreicht hatte. Jede weitere Selbstschwächung und innere Chaotik konnte nach Meinung dieser Intellektuellen nur zur totalen Unterjochung des Reiches durch die Mächte führen und mußte deshalb um jeden Preis vermieden werden. Andererseits waren sich die "Revolutionsgegner" durchaus schmerzvoll der Tatsache bewußt, daß die steril-dogmatische Orthodoxie des Konfuzianismus und die intellektuelle Unbeweglichkeit der traditionellen Staatseliten und Herrschaftsträger mitentscheidend die Ohnmacht Chinas gegenüber dem Einbruch der Mächte verursacht hatten. Sie plädierten daher für ein System grundlegender Reformen, das zu einer psychologischen und geistigen Läuterung und Selbststärkung der chinesischen Gesellschaft führen sollte. Auf diesem Wege könnte dann die nationale "Wiederauferstehung" nachfolgen und die politischen Institutionen des neuen, nationalen China konstitutionell so gestärkt werden, daß sie den Bewegungsabläufen der inneren und äußeren Politik der modernen Welt gewachsen waren.

1898 gelang es dem Kreis der nationalen Reformer erstmals, beim Thron Gehör zu finden. In der berühmten „Hundert-Tage-Reform“ versuchten sie einige ihrer grundlegenden Ideen in die Praxis umzusetzen. Das Reformwerk scheiterte jedoch am Widerstand der orthodoxen Kräfte, die unter der Führung der Kaiserinwitwe Tz'u Hsi den jungen Kaiser de facto entmachteten. Diejenigen Führer

\footnotetext{
1 Dieser antagonistische Entwicklungsablauf zwischen isolierter wirtschaftlicher Modernisierung und soziopolitischer Zersetzung des historischen "China“-Bewußtseins fand seine ideologische Entsprechung in Mao Tse-tungs revolutionärer Stadt-Dorf-Theorie, wonach die städtischen Zitadellen der antirevolutionären Kräfte von den dörflichen Revolutionsmassen ständig enger eingeschnürt und schließlich als Fremdkörper Kräfte von den dörflichen Revolutionsmassen ständig enger eingeschnürt und schließlich als Fremdkörper China wurde diese Theorie auf die Weltsituation zwischen den modernen Industrienationen und der Masse der Entwicklungsländer ausgeweitet und zum ideologischen Leitmotiv der chinesischen Weltpolitik stilisiert.
} 
der Reformbewegung, die der folgenden Hinrichtungswelle nicht zum Opfer fielen, flohen nach Japan. Dort bildete sich nach kurzer Zeit in der verhältnismäßig freien Atmosphäre des Landes eine Bewegung heraus, die gestützt auf das Studium deutscher Staatslehren eine nationale Reformideologie entwickelte ${ }^{2}$ und in China ständig wachsenden Einfluß fand. Eine der entscheidenden politischen Persönlichkeiten und unumstrittener intellektueller Führer dieser Partei zur Bewahrung der Monarchie, Pao-Huang Tang, war der bekannte Gelehrte und Mitgestalter des gescheiterten Reformwerks von 1898 Liang Ch'i-ch'ao.

Eine Auseinandersetzung mit der Person Liang Ch'i-ch'aos und seinem Einfluß auf die politische Szene Chinas zu Beginn des 20. Jahrhunderts vermag von mehr als rein akademisch-historischem Interesse zu sein, denn unabhängig von den direkten Wirkungen seiner politischen Agitation auf die Politik verschiedener chinesischer Regierungen in den ersten Jahrzehnten der Republik, 1911-1949, repräsentierten Liang und seine Anhänger ein nationales Gährungselement, das den Bewußtseinsboden für die revolutionären Bewegungen Chinas bis zur Gründung der Volksrepublik darstellte. Und selbst bis heute konstituieren ihre Ideen und Aufrufe zur politischen Zukunft Chinas eine Größe, die nicht ohne weiteres zu vernachlässigen ist, wie die ständige Auseinandersetzung der kommunistischen Partei mit der "bourgeoisen Linie“ der Intelligenz beweist. Diese Auseinandersetzung reicht bis in die Sphäre der polemischen Terminologie hinein; denn noch während der Kulturrevolution wurden die intellektuellen Führer, die zu Zielscheiben der rotgardistischen Angriffe geworden waren, häufig als „Pao-huang Tang" bezeichnet, also mit dem Namen jener Partei, deren geistiger und politischer Führer Liang Ch'i-ch'ao war. Andererseits hat kein Geringerer als Mao Tse-tung selbst in seinen Schriften hervorgehoben, wie sehr seine politische und revolutionäre Persönlichkeit von den frühen, national gesonnenen Führern der Intelligenz, insbesondere von den Vorstellungen und Appellen Liang Ch'i-ch'aos, entwickelt und mitgeprägt worden ist. Vor allem das populistische Erbe in Maos Denken, der Ursprung seines revolutionären Dranges, die Würde und Größe des chinesischen Volkes vor sich selbst und der Welt wiederherzustellen, ist mitentscheidend auf den politischen Einfluß Liangs zurückzuführen. Dies ist ein Faktor, der unter anderem von der Sowjetführung in ihrer politisch-ideologischen Polemik mit den chinesischen Kommunisten ausgenutzt wird als Zeugnis für die „chauvinistische Grundnatur" der Maoisten hinter ihrer „sozialistischen Fassade“.

Liang Ch'i-ch'ao war zweifelsohne einer der liberalsten Repräsentanten unter den modernen, chinesischen Intellektuellen, wenn sein Lebenswerk von persönlichen Idealvorstellungen her beurteilt wird. Ein Großteil seiner Schriften ist von hoher Bewunderung für Locke, Bentham, John Stuart Mill, Rousseau und andere Vertreter der westlichen Demokratiegeschichte durchwirkt. Andererseits jedoch betonte Liang selbst wiederholt - insbesondere in einer seiner Zentralschriften "Über den aufgeklärten Absolutismus" —, daß diese "subjektiv“ höherwertigen

2 Japan hatte sich in den vier Jahrzehnten "konservativer “ Modernisierung seit der Meiji-Reform von 1868 im staatlich-politischen Bereich weitgehend auf preußisch-deutsche Lehrvorbilder gestützt, und Staatsrechtsgelehrte wie Lorenz von Stein, Rudolph von Gneist und Hermann Roesler hatten maßgeblichen Anteil an der Gestaltung der Meiji-Verfassung. Aufgrund der japanischen Ubersetzungen fand die chinesische politische Intelligenz freien Zugang zu deutschen Staats- und Gesellschaftslehren des späteren 19 . und frühen 20. Jahrhunderts. 
Ideale gesellschaftlichen Lebens für die „objektive“ Bewältigung der zeitgeschichtlichen Problematik Chinas ungeeignet und illusorisch waren ${ }^{3}$.

Liangs evolutionäres Reformbewußtsein muß um so erstaunlicher scheinen, als er in den Jahren 1899-1902 nach dem Staatsstreich der Kaiserinwitwe und der Flucht nach Japan durchaus nicht immer der konsequente Reformideologe und „Revolutionsgegner" war, zu dem er nach 1902 werden sollte.

„Ich sehnte mich seit Jahren nach dem republikanischen System. Ein Teil der entschlossenen und patriotischen Männer im Lande sehnt sich ebenfalls in Úbereinstimmung mit mir seit Jahren nach dem republikanischen System. Wenn ich jedoch jetzt die Argumente der beiden Gelehrten Bluntschli und Bornhak lese, so ist das wie ein Guß kalten Wassers auf den Rücken. Mit einem Mal habe ich meine Grundprinzipien völlig verloren und bin voller Unsicherheit und weiß nicht mehr, welchen Weg man noch begehen kann. Gemäß den Aussagen der beiden Gelehrten sind die Eigenschaften, die ein Volk in der Republik besitzen sollte, in unserem Volke nirgendwo gegeben, und die durch die Geschichte überbrachten Gewohnheiten stehen zu jenem (System) geradezu im Gegensatz. Das ist etwas, was wir nicht verschweigen dürfen. Wenn wir es jetzt gewaltsam einführen wollen, werden wir nicht erfolgreich sein. Sollten wir es aber erreichen, werden wir dann aus Frankreich und Südamerika eine Lehre ziehen? Was uns deren Geschichte erzählt, wie sehr müssen wir das fürchten!... Was das Glück betrifft, wo bleibt da Euer Vaterland? Ach! Die Republik, von der ich seit Jahren träume, nach der ich mich sehne, und die ich besungen habe, hat mich schließlich verlassen... Ach! Republik, ich liebe dich! Aber ich liebe dich nicht so sehr wie das Vaterland!" (a) ${ }^{4}$

Nachdem Liang Ch'i-ch'ao erkannt hatte, daß die gesellschaftlichen und politischen Voraussetzungen für ein demokratisch-republikanisches System in China historisch und psychologisch nicht gegeben waren, lehnte er es trotz persönlicher Präferenz entschieden als staatliche Lösungsmöglichkeit ab. Liang hatte damit seine geistige Größe und intellektuelle Konsequenz als nationaler Theoretiker sowie sein Verständnis für die Verhältnismäßigkeit geschichtlicher Entwicklungsgrenzen deutlich gemacht. Im Gegensatz zu seinen ikonoklastischen Kontrahenten, der „Revolutionsallianz" um Sun Yat-sen, ließ er sich sein Urteil nicht durch politisches Wunschdenken trüben ${ }^{5}$.

Es ist daher keineswegs verwunderlich, wenn Liang in seiner Analyse der Staatsformlehre das Prinzip der nationalen Monarchie bevorzugte, das auch institutionell das angestrebte Ziel einer einheitlich-souveränen Staatsgewalt sichtbar machte. Liang ging dabei von Bluntschlis Untersuchung über den Charakter und Wert

\footnotetext{
3 Im Rahmen dieses Artikels können die grundsätzlichen Parallelitäten der geschichtlichen Entwicklung des Nationalismus in Deutschland und Japan einerseits und der historischen Situation Chinas zu Ende des 19. Jahrhunderts andererseits nicht abgehandelt werden. Es mag der Hinweis genügen, daß der Nationalismus in diesen Ländern eher eine ethnisch-psychologische Reaktion auf die expansiven Außerungen eines fremden Nationalismus war, - napoleonischer Nationalismus bzw. Imperialismus der westlichen Mächte - als der ideologische Ausdruck einer gesellschaftlichen und wirtschaftlichen Entwicklungsfolge, die die nationalstaatliche Integration der betroffenen Gesellschaften historisch erzwang. Aus dieser Parallelität resultierte auch die besondere Anziehungskraft der deutschen und japanischen Vorbilder für die chinesischen Intellektuellen.

4 Die Quellenangaben für die chinesischen Texte werden in alphabetischer Form gegeben und am Ende des Artikels angeführt.

5 Daß Liangs Alternative einer Monarchie, in der die Macht stufenweise von der autokratischen Herrschaft auf die Gesellschaft übergehen sollte, sinnvoller war als die Radikalität der Revolutionäre, beweist die Situation nach der Revolution von 1911. In diesem Zeitraum wurden alle die Phänomena sichtbar, vor denen Liang gewarnt hatte. Das soziale und politische Chaos des "Warlordism “ in den ersten Jahrzehnten der Republik rechtfertigten ihn nachträglich, und die heutige Herrschaftsform in der Volksrepublik ist ebenfalls weit von den Vorstellungen der frühen Revolutionäre entfernt.
} 
der demokratisch-republikanischen Politik aus ${ }^{6}$, um sich dann auf die systematische Abhandlung der Staatsformen in Conrad Bornhaks „Allgemeiner Staatslehre" zu beziehen ${ }^{7}$ und sich positiv für die "Verfassungsmonarchie" als Staatsform Chinas zu entscheiden ${ }^{8}$.

Im Bewußtsein Liangs konnte die nationale Modernisierung Chinas nur als ein Generationenwerk verstanden werden, in dem sich das chinesische Volk selbst und seine gesellschaftlich-wirtschaftlichen Existenzbedingungen grundlegend umgestaltete., Erst wenn ein solcher Reformprozeß durchgeführt war, konnte man sich institutionell-politischen Fragen zuwenden. Unter Auslassung dieser Vorbedingungen erschien eine republikanische Staatsform wenig sinnvoll und in ihren Folgen gefährlich.

Diese Argumentation Liangs wurde durch Bluntschlis Thesen zur republikanischen Staatsform bestärkt. Danach mußte eine Gesellschaft die „republikanischen Tugenden" in hohem Maße internalisiert haben und auf einem angemessenen, politischen Erziehungsniveau stehen, wenn sie republikanische Politik sinnvoll ausüben wollte. Ein Volk, das diese lange intellektuelle und politische Reife nicht mehr oder noch nicht besitzt, wäre in einer Republik zum politischen Zerfall verurteilt und müßte zur Diktatur hin degenerieren. Die intellektuelle und politische Reife konnte ein Volk jedoch nur über viele Generationen hinweg graduell anstreben, wie es in den USA der Fall gewesen war. Dort hatten die Bürger auf eine lange, angelsächsische Tradition zurückblicken können, als sie die Unabhängigkeit erreichten. Eine Republik durch politischen Umsturz des alten Staatswesens, durch eine Revolution, verwirklichen zu wollen, das dürfte in der Regel zu negativen Ergebnissen führen. Die ersehnte Republik endete in der Terrorherrschaft der Nachrevolution. Die Geschichte Frankreichs und der südamerikanischen Länder diente hierfür als mahnendes Beispiel. Die politischen Ideale führten zu anarchischem Chaos und schließlich zur Diktatur. Die betroffenen Länder waren also von einem demokratischen Status trotz aller Anstrengugen noch mehr oder weniger weit entfernt, oder sie hatten ihn nur unter großen Leiden und Wirren erreicht. Von diesen Aussagen Bluntschlis her ging Liang zu Bornhaks Theorie über.

„Man kann die Argumentation Bluntschlis über den Wert des republikanischen Systems als genau und tief bezeichnen, aber sie ist noch nicht vollkommen. Wenn man einmal die Staatslehre des deutschen Gelehrten an der Universität Berlin, Conrad Bornhak, liest, so wird das in folgenden ausreichend dargestellt. Das Original wurde 1896 herausgegeben und ins Japanische übersetzt. Es ist in der Tat die neueste Lehrmeinung. Bornhak fragt: Was ist der Staat? Er ist eine Vereinigung zur Abwägung des Rechten und zum Ausgleich aller Arten von Interessen und Widersprüchen innerhalb der Gesellschaft (b)."

Conrad Bornhak hatte also die Lehrformel für den Staat geprägt, die Liang als geeignet und nützlich für die chinesische Sphäre ansah. Der Staat als autoritative Quelle der Gerechtigkeit und des Konfliktausgleichs im Innern und als Schutzorganisation gegenüber anderen Volksgemeinschaften war nahezu vollkommen das-

6 Johann, Caspar Bluntschli, Lehre vom modernen Staat, Bd. I, Allgemeine Staatslehre, 6. Buch, Die Staatsformen, Stuttgart, 1875.

7 Conrad Bornhak, Allgemeine Staatslehre, Berlin, 1896 . tionell-parlamentarischen Monarchie herzustellen und eine Monarchie preußisch-deutschen und japanischen Vorbilds zu beschreiben. Auf eine genauere Definition wird an späterer Stelle noch eingegangen. 
jenige System, das Liang zur Realisierung seines Reformnationalismus vordringlich benötigte und sich daher zu eigen machen wollte.

Einem solchen Staatssinn unter den chinesischen Vorbedingungen mit der Errichtung einer demokratischen Republik nachkommen zu können, hielt Liang für schlechthin unmöglich. Die Republik wies seiner Meinung nach bedeutsame Strukturschwächen auf, die er nach dem Studium der Bornhakschen Staatslehre kommentierend hervorhob.

„Bornhak sagt weiter, daß die Erlangung des republikanischen Systems durch Gewohnheit in der Regel gesichert sei und daß die Erlangung des republikanischen Systems durch eine Revolution in der Regel gefährdet sei. Ich möchte über diesen Grundsatz sprechen. Wenn man durch die Gewalt der Revolution die alten und überlieferten Staatsverfassungen hinwegwischt und das höchste Ziel des Staates auf die lastvollen Schultern des Volkes legt, und wenn man den Aufruhr hinnimmt, um dann durch heftige Parteizwiste das gespaltene Volk veranlassen $\mathrm{zu}$ wollen, das Gleichgewicht gesellschaftlicher Einflüsse zu bewahren, dann ist auch das eine Rechnung, die nicht aufgeht (c)."

Eine gesellschaftliche und politische Entwicklung zum staatlichen Chaos und zur Instabilität hin widersprach der nationalen Zielsetzung Liang Ch'i-ch'aos, die ja gerade darin beruhte, eben diesen Niedergang in China aufzuhalten und rückgängig zu machen.

Liang erklärte sich die Schwäche Chinas vor allem aus der geringen Ausbreitung seines Staatsbewußtseins, das sich in einem Mangel an Patriotismus negativ bemerkbar machte. Dieser Mangel hatte dazu geführt, daß das Reich sich allmählich zu einer "Privatautokratie“ entwickelt hatte, und hierin lag nach Liangs Auffassung auch die eigentliche Ursache für den Geltungsverlust Chinas und für die Bedrohung seiner Souveränität. Vor einem solchen Schicksal konnte nur eine nationale Erneuerung bewahren.

„Nach den Reformen zur Selbststärkung (in Japan) und nach der Revision der Verträge (zwischen Japan und den westlichen Staaten) war die Staatssouveränität (Kuo-ch'üan) schließlich vollständig unbeeinträchtigt. Wenn sich unser Volk daher diese Umstände betrachtet, dann muß patriotische Glut die Brust erfüllen und sich ausbreiten, so daß niemand sie auszutreten vermag (d).“ "Wissen sie (orthodoxe Führer Chinas) etwa nicht, daß der sogenannte Fort. schritt zur heutigen Vorherrschaft der Länder England, Frankreich, Deutschland und USA nur eine Angelegenheit von hundert und einigen Jahren war? Und das, wodurch sie zum Fortschritt befähigt wurden, hat kein anderes Geheimnis als nur das, daß die Menschen eines Landes die Angelegenheiten des Landes in die Hand nahmen und daß das Land nicht Privatbesitz des Herrschers, sondern Gemeinbesitz des Volkes war (e).“

Unter diesem Gesichtspunkt müssen daher auch die Beschaffenheit und Aufgabenstellung des Staates, also der Staatssinn, untersucht werden. Der Staat hatte seine Entstehungsursachen für Liang Ch'i-ch'ao ausschließlich in der Konfrontation mit anderen Gemeinschaften und dem daraus resultierenden Willen zur Selbstbehauptung und Souveränität.

„Gemäß dem allgemeinen Gesetz des Wettstreits der Dinge und der natürlichen Auslese müssen die Menschen aufeinanderstoßen, müssen die Länder aufeinanderstoßen. Den Begriff Staat stellte man auf, um auf andere Gemeinschaften zu reagieren. Deshalb will kein wahrer Patriot, selbst wenn es in fremden Ländern große Heilige und Weise gäbe, sich unter deren Herr- 
schaft fügen. Wäre es nicht besser, das Blut aller Menschen im Lande zu vergießen und die Körper zu verstreuen, bis niemand übrigbleibt, als auch nur das geringste Recht an andere Rassen abzutreten? Wenn das nicht so ist, dann bereitet man dadurch mehr als alles andere den Untergang des Landes vor (f).“ Seiner Entstehungsursache und Aufgabenstellung, der Erhaltung der nationalen Souveränität, konnte der Staat jedoch nur nachkommen, wenn er durch einen autoritativen Ausgleich im Innern die Einheit des Staatsvolkes nach außen hin absicherte. Diese Bedeutung des Staates für die nationale Souveränität verschaffte ihm als handelndem Teil des organischen Verhältnisses eine gewisse Vorrangigkeit gegenüber dem Volk.

„Das am Staat Schätzenswerte ist, daß er eine Herrschaft hat (Yu-chih), mit der er im Innern die Konkurrenzkämpfe ausgleichen und mit der er nach außen hin die Konkurrenzkämpfe unterstützen kann. Beides ist in der Tat voneinander abhängig in seinem Nutzen. So kann man in einem Satz sagen, daß der Staat den Geist für die Errichtung der Herrschaft darstellt. Wenn die äußere Form (der Herrschaft) diesen Geist achtet, dann kann das als gut bewertet werden. Wenn die äußere Form sich gegen diesen Geist wendet, dann kann man das als schlecht bewerten (g).“

„Die Gelehrten des 19. Jahrhunderts sagen, daß der Staat (Kuo-chia) sicherlich im Interesse des Volkes (Kuo-min) besteht, aber gleichzeitig das Volk auch im Interesse des Staates. Der Staat plant einerseits den Vorteil des Volkes, andererseits aber für sich selbst. Wenn der Gewinn des Volkes und der des Staates nicht übereinstimmen, dann hat der Gewinn des Staates Vorrang, und der Gewinn des Volkes steht hintenan. So ist der Staat ein Ziel in sich, und das Volk kann bisweilen ein Mittel sein, um zu diesem Ziel beizutragen $(\mathrm{h})$."

Liang Ch'i-cha'os reformerischer Staatsbegriff konnte nicht angelsächsisch geprägt sein; denn der Staat sollte nicht nur einen Teilbereich der liberalgesellschaftlichen Komplexität ausfüllen, sondern eine institutionalisierte Form der Gesellschaft schlechthin darstellen. Er mußte sich von allen anderen sozialen Gruppierungen dadurch abheben, daß er mit der ausschließlichen und höchsten, ausübenden Gewalt versehen war. Er sollte ferner den "Volkswillen“ organisatorisch erfassen und durch die Realisierung der nationalen Gemeininteressen politisch sichtbar machen.

Die Regierung des Staates konnte folglich weder außerhalb noch oberhalb des Staatswesens eingesetzt werden. Sie hatte vielmehr den Mechanismus zu bilden, durch den der Staat aktive Herrschaft ausübte und die gesetzliche wie moralische Autorität kontrollierend wirksam machte.

Liangs Staatsbegriff stand also im Zeichen einer Gewalt, die von der Staatswissenschaft mit dem Begriff "Souveränität" bezeichnet und die im Innern wie nach außen hin als höchste, umfassende und nicht mehr ableitbare Gewalt verstanden wird. Den Anspruch hierauf rechtfertigte Liang durch eine aus der chinesischen Notsituation resultierende, übergeordnete Staatsnotwendigkeit, durch seine Theorie der Staatsräson.

Der Souveränitätsanspruch des im zwischenstaatlichen Bereich nur sich selbst und seinem Wohl verpflichteten Staatswesens galt nach Liangs Auffassung als das eigentliche Merkmal für die Reife nationaler Staaten. Aus diesem Grund ist es unerläßlich, festzuhalten, welche Kriterien Liang als notwendig für die Souveränitätsqualifikation Chinas ansah. Vorweg sei gesagt, daß er die Rousseauische Lehre als un- 
brauchbar für Chinas Staatsmodell ablehnte, da Rousseau durch seine Identifikation des allgemeinen Volkswillens mit dem Souveränitätsprinzip letzten Endes auf eine Synthese der demokratischen Gesellschaft und der staatlichen Hoheit ausgerichtet war. Eine solche Synthese aber hätte nach Liangs Meinung in der chinesischen Sphäre unweigerlich zu einem permanenten Spannungsverhältnis der verschiedenen sozialen Gruppierungen führen müssen.

„Rousseau sagt, daß die Souveränität (Chu-ch'üan) nicht beim Herrscher, sondern beim Volke liege. Der Gesamtwille des Volkes ist der Souverän... Wenn man sagt, daß die Souveränität nicht beim Herrscher liege, sondern bei der Volksgesamtheit, dann wird die Souveränität niemals ausgedrückt, da die Meinung des ganzen Volkes nie in Einklang gebracht werden kann und erfaßt werden kann. Daher könnte ein Teil des Volkes trügerisch sagen, daß seine Meinung die der Gesamtheit sei und so die Souveränität verletzen. Daraus entsteht das große Unglück einer Revolution. Die Absichten des Volkes ändern sich oft und sind instabil (i)."

Der Staat als Quelle der Gerechtigkeit und sozialen Harmonie bedurfte eines weniger metaphysischen Souveränitätskonzeptes, als Rousseau es anzubieten wußte. Souveränität mußte eine deutlich abgegrenzte, gesetzliche und moralische Autorität verkörpern, die entscheidende Richtlinien setzen konnte und keinerlei gemeinschaftsgefährdende Konflikte tolerierte. So stellte sich die souveräne Hoheitsgewalt des Staates als ein Phänomen dar, das sich durch fünf Grundkriterien definieren ließ.

Erstens, unableitbare Unabhängigkeit des Staates;

zweitens, Unterordnung aller Institutionen und Organisationen unter die staatliche Herrschaft;

drittens, Übergeordnetsein über allen Machtorganen im Staat, viertens, Unteilbarkeit der Gewalt;

fünftens, als tatsächliche Wirksamkeit ist die Souveränität begrenzt, da die Staatsführung als Souverän dem Diktat der Verfassung als der Quelle der Souveränität unterliegt.

Letzter Ausdruck der Souveränität war also das Gesetzessystem, über das sich die Herrschaft nicht hinwegsetzen durfte. Die Autorität des Gesetzes bildete den Urgrund wahren staatlichen Seins in der Lehre Liang Ch'i-ch'aos.

„Die Souveränität (Chu-ch'üan) gehört nicht allein dem Herrscher und nicht allein der Gesellschaft. Sie ist weder oberhalb noch außerhalb des Staates. Die Existenz des Staates und die aus ihm festgelegte Verfassung sind das, woraus die Souveränität hervorgeht. Manche sagen, daß die Gesellschaft eine Vereinigung von Individuen sei. Die Souveränität wäre also die vereinte Autorität der Individuen. Diese Worte sind in höchstem Maße irrig. Die Souveränität ist ein öffentliches Recht (Kung-ch'üan) und kein Privatrecht (Ssu-ch'üan). Selbst die Summe unzähliger Privatrechte kann nicht deren Natur zu einem öffentlichen Recht umwandeln (j)."

„Manche sagen, daß nach der Vereinigung einer Nation, selbst wenn sie noch keinen Staatskörper (Kuo-chia-t'i) hat, dennoch von einer Souveränität gesprochen werden könne. Diese These ist falsch. Eine Nation kann keine juristische Person sein. Ohne das Vorhandensein einer Gestalt kann es nicht schon einen Geist geben. Deshalb gibt es Souveränität, wenn es einen Staat gibt. Wenn es keinen Staat gibt, dann gibt es auch keine Souveränität (k).“ 
Unter Souveränität verstand Liang also die durch das Verfassungsrecht eingegrenzte, staatliche Autorität und Gewalt, die die Regierung als Träger der Staatsherrschaft im Interesse des Staatsvolkes ausübte. Dieses Interesse des Volkes sollte von der ideologisch bewußten Herrschaft selbst bestimmt werden, da das chinesische Volk aufgrund seiner autokratischen Vergangenheit nicht die politische Reife besaß, um aus der staatlichen Vormundschaft entlassen zu werden.

Die gesamte Souveränitätstheorie Liangs, wie sie im vorangehenden Abschnitt dargelegt worden ist, stützte sich weitgehend auf Bluntschlis Lehre vom modernen Staat. Insbesondere folgte er Bluntschli in der Ablehnung der Gesellschaftsvertragstheorie Rousseaus, da sie seinem Hauptanliegen, der Stärkung des Staates für die Konfrontation mit anderen Staaten, entgegenlief. Rousseaus Versuch, seine philosophische Souveränitätstheorie durch eine „Funktionstheorie“ zu ersetzen, indem er den Willen der Mehrheit mit dem der Gesamtheit für praktische Zwecke gleichsetzen wollte, erschien ihm als unglaubwürdig.

„Nach den Argumenten Bluntschlis paßt der Gesellschaftsvertrag (Min-yüeh lun) zur Gesellschaft (She-hui) und nicht zum Staat (Kuo-chia). Wenn man ihn sorglos darstellt, dann wird das Volk (Kuo-min) wieder in Splitterbevölkerungen (Pu-min) zerfallen, und man vermag die Splitterbevölkerungen nicht zu einem Volk zu verschmelzen. Sicherlich hat diese Lehre (vom Gesellschaftsvertrag) ihre Wirksamkeit gehabt als eine Medizin gegen die unmäßigen Übergriffe im damaligen Europa. Wenn man sie aber auf das auseinanderfallende China überträgt, dann weiß ich nicht, ob die Nachteile und Vorteile einander auszugleichen vermögen... Was die häufig mißbrauchten Worte "Freiheit" und "Gleichheit" angeht, so sind die Ideen dazu übersteigert (in China), und die Voraussetzungen reichen nicht aus, um sie abzustützen ... Alle von Bluntschli bei Rousseau aufgezeigten Mängel bereiten heutzutage die neuen Denker bei uns wieder vor... Ich wage es nicht, Bluntschli hervorzuheben und Rousseau zu erniedrigen. Nur meine ich, daß diese große Lehre, die einen Angriff auf (Rousseaus) Lehre darstellt, von jemandem, der den Staat aufrechterhalten will, immer wieder in Betracht gezogen werden sollte (1)."

Nachdem Bluntschli die naturrechtliche Erörterung vom Wesen und den Ursachen des Staates und seiner „Vertragsgesellschaft" als historisch nicht verifizierbare Spekulation verworfen hatte, begründete er die Staatsursache und das Staatsziel in seiner Organismustheorie, wonach der Staat gleichsam eine Person mit eigenem Geist und Körper darstellte und einen eigenen Willen sowie eine selbständige Handlungsbewußtheit besaß.

Liang stimmte den Ansichten Bluntschlis über die Entstehung und das geschichtliche Wesen von Staaten und Völkern als einer organischen Einheit zu und übernahm sie für sein chinesisches Modell.

„Wenn der Staat einen Organismus (Chi-t'i) hat, dann kann etwas, das als Organismus nicht vollendet ist, auch nicht als Staat bezeichnet werden. China ist dann ein verkrüppelter und kranker Organismus. Es ist ebenfalls richtig, daß es kein (richtiger) Staat ist $(\mathrm{m}) .{ }^{\text {“ }}$

„Das Volk ist eine Persönlichkeit, die den organischen Staat (Yu-chi chih kuochia) als Körper (T’i) besitzt und die ihr Denken (Yi-hsiang) äußern und ihre Rechte festhalten kann. Zweitens ist das Volk eine gesetzliche Gemeinschaft (Fa-t'uan), die im Gesetzeskörper (Fa-lü-t'i) des Staates existiert. Der Staat ist für alle Zeit eine vollkommene, geeinte Körperschaft öffentlicher Art, 
die sich auf den Geist des Volkes (Kuo-min chih ching-shen) stützen muß, um sich selbst zu erfüllen. Erst dann ist ein vollendeter Zustand erreicht. Wenn es daher ein Volk gibt, dann gibt es auch einen Staat. Wenn es keinen Staat gibt, dann gibt es auch kein Volk. Beide sind in der Tat eine Sache mit zwei Namen. Da die Nationen (Min-tsu) gleiche Sprache, gleiche Sitten, gleichen Geist und Charakter haben, entwickelt sich das Gemeinbewußtsein allmählich. Das ist sicherlich eine Stufe zum Aufbau des Staates. Aber in der Zeit, da man sich noch nicht zu einem Staat vereint hat, kann man unter keinen Umständen eine Persönlichkeit und eine gesetzliche Körperschaft bilden. So kann nur die Bezeichnung Nation und nicht die Bezeichnung Volk verwendet werden (n).“

Liangs Reformnationalismus war also auf das Ziel einer chinesischen Nation ausgerichtet, die ihrer Bestimmung zum „Volksstaat“ bewußt gerecht werden sollte. Das Verhältnis des chinesischen Volkes zu diesem neuen Staat sah Liang in seinem Souveränitätsprinzip festgelegt, wonach das staatliche Gesetz die Manifestation der Souveränität sei und die staatliche Herrschaft den konkretisierten Souverän darstelle. Obwohl zwischen der unbegrenzten, ideellen Souveränität des Staates und der begrenzten, konkreten Souveränität der Herrschaft unterschieden werden müsse, bildeten beide im Normalfall ein Harmonieverhältnis, wie es zwischen Haupt und Gliedern eines Organismus bestände. Ein Konflikt zwischen der Staatssouveränität als gesetzlicher Gewalt und der Herrschaftssouveränität als regierender Gewalt erschiene vom Prinzip her unmöglich, da es sich dann um einen Konflikt zwischen dem „Haupt“ und dem „Haupt plus Körper" handele, also um einen Konflikt der gleichen Person mit sich selbst. Liangs Staatstheorem richtete sich also auf eine zentralisierte Staatsgewalt hin aus, in der alle sozialen Gruppierungen der chinesischen Gesellschaft einem Organismus gleich integriert und zugeordnet waren. Bluntschlis Lehre erschien ihm dabei von besonderem Nutzen und besonderer Bedeutung zu sein.

„Rousseau, John Stuart Mill, Spencer, alle diese Gelehrten werden nicht mehr beachtet. Selbst das die Freiheit liebende Amerika konnte nicht umhin, seine Ziele zu ändern und die Macht in der Zentrale zu konzentrieren und den Einfluß der Regierung zu erweitern, um nach außen hin zu kämpfen. Über die anderen Länder braucht man gar nicht erst zu reden. Die drückende Tendenz der allgemeinen Lage und ihre Einwirkung hängen sicherlich nicht von ein oder zwei Leuten allein ab, aber ist der Einfluß der Ideen auf die Realität nicht groß? Wenn man sagt, daß Rousseau der Vater des 19. Jahrhunderts sei, dann ist Bluntschli der Vater des 20. Jahrhunderts (o).“

Institutionell und vom Staatsformverständnis her sollte der chinesische Staatsorganismus durch eine nationale „Verfassungsmonarchie“ repräsentiert werden, d. h. durch eine konstitutionelle Monarchie, in der das staatliche Leben durch das monarchistische Prinzip bestimmt wurde, so wie es in Deutschland durch Friedrich Julius Stahl eine Sinngebung erfahren hatte.

Liang Ch'i-ch'ao betrachtete in Übereinstimmung mit der deutschen Anlage die folgenden Minimalkriterien als unerläßlich für eine „Verfassungsmonarchie“.

Erstens, der Monarch steht in einer verfassungsmäßigen Würde und Macht;

zweitens, der Monarch übt seine Herrschaft verfassungskonform aus;

drittens, die monarchische Gesetzgebungsgewalt unterliegt einer Mitwirkung und Zustimmung des Parlaments. Initiative und Sanktion verbleiben jedoch beim Monarchen; 
viertens, der Monarch vermag nur mit ministerieller Beteiligung und Einvernahme rechtswirksam Regierungshandlungen durchzuführen;

fünftens, die Rechtsprechung hat einen eigenständigen Charakter und muß eine Kabinettsjustiz ausschließen;

sechstens, Staatshaushalt und Steuerwesen werden durch das Parlament kontrolliert und abgegrenzt.

Aufgrund dieser Vorbedingungen verstand Liang die „Verfassungsmonarchie“ als die Monarchie eines „freien Volkes“. So glaubte er, dieser Staatsform die Beschreibung "Volksmonarchie“ insofern zuerkennen zu dürfen, als in ihr der Monarch die Personifikation des wahren Staatswillens ausmachte und nicht bloß als ein formales Zusatzelement der parlamentarischen Herrschaft und des Eigenwillens der politischen Mehrheitsgruppierung anzusehen war. Die Herrschaft erfolgte demgemäß zwar nicht durch das Volk, aber im "wahren Geist" und Vertrauen des Volkes zum „wahren Wohl“ des Volkes. Die „Verfassungsmonarchie“ mußte daher als die dem Volk in seiner Gesamtheit angemessenste und vollkommenste Staatsform akzeptiert werden. Entscheidendes Moment der „Verfassungsmonarchie“ war nach Liangs Meinung, daß in ihr die soziale Vielfalt in harmonischer VerhältnismäBigkeit ausgerichtet war und die Einheitlichkeit der Staatsgesamtheit gewahrt wurde. Der Wille zur Einheit als die höchste aller nationalen Qualifikationen fand somit in der "Verfassungsmonarchie" seine Institutionalisierung; denn das Verfassungswesen der "Verfassungsmonarchie" berücksichtigte vor allem den Geist der "nationalen Repräsentation" und die Einigkeitsdarstellung des Volksstaates. Erst in zweiter Linie betonte es die sozialen Sonderinteressen. Im Hinblick auf eine derartige "nationale“ Schwerpunktsetzung konnte das Parlament nur eine komplementäre Funktion ausüben und eine Position einnehmen, deren Hauptzweck in der Erhöhung des Öffentlichkeitsbewußtsein und in einem Abbau gegensätzlicher Gesellschaftsvorstellungen lag. Es fungierte also als ein Staatsorgan mit mehr moralischrechtlicher Einwirkung auf das Volksganze und übte weniger direkte, politische Macht aus.

Liangs Staatstheorem, konzipiert als nationale Kampforganisation, besaß keinerlei Verwendungsmöglichkeiten für ein dysfunktionales Element in Form parlamentarischer Parteiherrschaft.

„Der Monarch steht über allen Arten von Interessenbeziehungen. Er repräsentiert den Staat als Großvereinigung, um sie in Einklang zu bringen. Das Schätzenswerte am Staat und das höchste Ziel des Staates sind hierin begründet. Die Schwierigkeiten zur Erlangung dieses Ziels sind in Republiken und Monarchien von unterschiedlicher Größe (p).“

"Ob jedoch dieser Maßstab (,daß eine parlamentarische Herrschaft die beste sei') richtig ist oder nicht, das ist sicherlich nicht einfach zu beantworten. Sind die Länder Südamerikas nicht Herrschaftssysteme, beruhend auf einer allgemeinen Gesetzgebung des Volkswillens? Welcherart nun ist der Gewinn für die Länder und das Glück für die Völker? Weiterhin, als Preußen und Óstereich einander den Krieg erklärten, wandte sich das preußische Parlament in seiner Gesamtheit gegen Bismarcks Politik. War das nicht der allgemeine Wille des Volkes? Wie aber konnte sich das mit der Politik Bismarcks messen, hinsichtlich des Gewinns für den Staat und des Glücks für das Volk (q)?“

„In allen konstitutionellen Staaten auf der Welt gibt es Zusammenstöße zwischen dem Parlament und der Regierung. Das kann noch nicht als ein Mißstand angesehen werden, aber es hängt davon ab, welcherart die Zusammen- 
stöße sind. Wenn beide fortwährend in Konflikt miteinander liegen, so daß es keine ruhige Zeit mehr gibt, dann kann das nicht der Grundwille des Konstitutionalismus sein ( $r)$."

Es hätte also Liangs Erkenntnis nach der Natur der „Verfassungsmonarchie“ widersprochen, das Parlament als Träger der Souveränität und staatlichen Hoheit zu verstehen. Er sah in ihm vielmehr ein verfassungsbedingtes Staatsorgan, dem die moralisch-rechtliche Repräsentation des Volkes und das Gesetzgebungskomplement für den Monarchen zugewiesen war. Diese parlamentarische Position wurde überschattet durch den Monarchen, für den die Personifizierung der Souveränität wesenhaft war. Die Souveränität war also nicht in dem Sinn geteilt, daß die anderen Staatsorgane wie das Parlament ausschließliche Rechte und Pflichten hatten, sondern nur durch die Verfassung eingeschränkt, die diesen Staatsorganen eine konkurrierende Rechtsposition vermittelt hatte. Eigentlicher Ruhepunkt der Souveränität war die Staatsverfassung, die den Monarchen als den physischen Träger der Souveränität einsetzte. Diese Ansicht Liangs beruhte auf Bluntschlis Aussagen in seiner modernen Staatslehre.

„Daher tadelt Bluntschli Rousseau entschlossen. Von hier ausgehend zeigt er die Quellen der Souveränität wie folgt auf: Die Souveränität gehört nicht allein dem Monarchen, aber auch nicht ausschließlich der Gesellschaft. Sie ist nicht oberhalb des Staates und nicht außerhalb des Staates. Die Existenz des Staates und die aus ihm festgelegte Verfassung sind das, woraus die Souveränität hervorgeht (s).“

Daß der Monarch als Träger der Souveränität bestimmt war und sie in keiner Weise mit den anderen Organen im Staat zu teilen hatte, erklärte sich aus der historischen Entwicklung der „Verfassungsmonarchien“. In ihnen hatte nicht ein gewachsenes Parlament eine den Herrscher einschränkende Verfassung erzwungen, sondern der Monarch selbst hatte sie durch seinen Willen erlassen. Erst mit dem Inkrafttreten dieser vom Monarchen erlassenen Verfassung wurde die Existenz eines Parlamentes überhaupt möglich.

Eigentlicher Staatswille war also der Wille des Monarchen, wenn er sich in den Grenzen der Verfassung als Handlungsgrundlage bewegte. Die Verfassung mußte daher als eine aus der Natur der Herrschaft selbst abgeleitete Erscheinung staatlichen Seins gelten. Der monarchische Wille als Staatswille konnte nur auf die Gemeininteressen ausgerichtet sein, ohne deren Erfüllung die eigentliche Staatsräson fortfiele. Diese aus der deutschen Tradition übernommene Theorie vom monarchischen Prinzip sollte als repräsentativer Ausdruck für Liangs Staatsmodell dienen, um das Bewußtsein chinesischer Zusammengehörigkeit und das staatsmoralische Pflichtdenken der chinesischen Öffentlichkeit zu erhöhen.

„Nun, wer sehnte sich nicht nach der Herrlichkeit der parlamentarischen Regierung? Aber es ist keineswegs so, daß es in Deutschland und Japan keine hervorragenden Staatsmänner und weise Gelehrte gäbe, und dennoch wollen sie diese allerschönste Politik nicht betreiben. Warum? Sie haben im Innern ihr Volk geprüft und erkannt, daß die Zeit dies noch nicht zuläßt... Wenn Preußen eine parlamentarische Politik ausgeübt hätte, dann hätte Bismarck zur Zeit des preußisch-österreichischen Krieges aus dem Amt scheiden müssen. Wie wäre das spätere, deutsche Kaiserreich dann wohl entstanden ( $t)$ ? “

„Die Grundzüge eines konstitutionellen Systems sind nicht einheitlich. Aber die Errichtung eines Parlamentes ist einer der wichtigsten Punkte dabei. Wenn jemand sagt, daß das Parlament unbedingt in einer aktiven Führungsrolle 
stehen muß, dann ist es von vornherein klar, daß das Niveau des Volkes dafür zu niedrig ist. In einer konstitutionellen Monarchie kann man das Parlament in eine Kontroll- und Hilfsposition stellen (u).“

„Das Parlament darf sein Anklagerecht nicht mißbrauchen. Wenn es angewandt wird, müssen richtige und unvermeidliche Gründe vorliegen. Dann ist es zwar gesetzlich auch nicht unbedingt nötig, Anklage und Verurteilung der Regierung vorzunehmen, aber in der Praxis führt es den Monarchen dahin, zu empfinden, daß eine Prüfung aufgrund der Gefahren für das Volk begründet ist, und es führt die Regierung dazu, vorsichtig zu sein und zu erkennen, daß die öffentliche Meinung von Bedeutung ist. Dann hat die Anklage, gleich ob die Regierung verdammt wird oder bestehen bleibt, immer eine Wirkung (v)."

Liang Ch'i-ch'ao sah die "Verfassungsmonarchie“ also als nutzvoll und realisierbar für China an. Sie war die geeignetste Herrschaftsorganisation für das nationalstaatliche Ziel des Konfliktausgleichs im Innern und des geschlossenen Zusammenstehens nach außen. Er wandte sich gegen eine republikanische oder „scheinmonarchische" Parlamentsherrschaft, weil diese nur zur Vertiefung und Intensivierung der sozialen und politischen Spaltungserscheinungen im Lande hätte führen können.

Dieser „verfassungsmonarchische" Staatsentwurf sollte sich in einer staatspolitischen Strukturgebung manifestieren, die auf eine "moderierte“ Gewaltausübung der Herrschaft hinzielte. „Moderation“ bedeutete für Liang die Vereitelung aller dysfunktionalen Regierungstätigkeit, d. h. aller solcher Handlungen, die einen Mißbrauch der Regierung zugunsten nichtstaatlicher Interessen verursachten, insbesondere den Amtsmißbrauch des Herrschers zu persönlichen Zwecken. Ein solches Fehlverhalten der Herrschaft wurde von Liang als "autokratisch" verurteilt. Aus den gleichen Gründen lehnte er eine absolute Verfügung der Staatsgewalt durch das Parlament in Form einer parlamentarischen Herrschaft für den chineischen Gebrauch $a b$, da ein parteipolitischer Mißbrauch der Regierung zuungunsten des Staatsganzen nur zu wahrscheinlich gewesen wäre.

"Die konstitutionelle Monarchie ist das beste Regierungssystem. Was die konstitutionelle Demokratie angeht, so ändert sich die politische Richtung zu oft. In der Zeit, da der Präsident gewählt wird, ist der Kampf zu heftig, und hinsichtlich des Staatswohls, gibt es immer abträgliche Einflüsse. In einer autokratischen Monarchie sieht der Hof das Volk als Gras an (über das der Herrscher wie ein Sturm hinwegfegt), und er hält es in Fesseln wie einen Räuber. Das Volk fürchtet den Hof wie einen Gefängnisaufseher und haßt ihn wie einen Todfeind. So unterliegt das Volk größter Pein, und Herrscher wie Staatsbeamte leben so in höchster Gefahr . . . Deshalb ist die konstitutionelle Monarchie das beste Regierungssystem. Wenn die Staaten auf der Erde sie aufrechterhalten, dann sind sie erfolgreich, und hinsichtlich der alten Sitten und der gegenwärtigen Situation Chinas bedeutet ihre Übernahme ebenfalls keinen Nachteil (w).“

Liangs Staatsmodell durfte weder die uneingeschränkte Souveränität des Herrschers noch die parlamentarische Souveränität im engeren Sinne tolerieren, wenn es sich nicht selbst in seiner Verwirklichung gefährden sollte. Es mußte sich vielmehr auf das Konzept der Volkssouveränität stützen. Wie jedoch bereits festgestellt worden ist, war unter dem Begriff Volkssouveränität eine deutsche, ganzheitliche Auslegung zu verstehen, im Gegensatz zur angelsächsischen Volkssouve- 
ränität parlamentarischer Prägung. Es handelt sich eher um ein politisches Prinzip, das als „innere Staatssouveränität" institutionell nur mittelbaren Ausdruck fand und in Lanigs Denken durch die Bluntschlische Identifikation von Staat und Volk verständlich wurde. Volkssouveränität in dieser Auslegung besagte nichts anderes, als daß alle staatlichen Organe, insbesondere der Herrscher, in demjenigen Rahmen politisch wirksam wurden, der ihnen durch die Staatsverfassung auferlegt war. Volkssouveränität konnte deshalb für Liang keinesfalls ein Synonym für demokratische Herrschaftsformen sein, sondern nur für verfassungskonformes Staatsleben. $\mathrm{Ob}$ die Verfassung selbst demokratische Organisationsformen begründete oder nicht, war dabei irrelevant für die Existenz des Liangschen Volkssouveränitätsprinzips.

Die Verfassung galt Liang als konkreter Lebensausdruck des Staatsorganismus, in dem sich kein Organ widernatürlich verhalten durfte und konnte, ohne das Leben des gesamten Organismus zu wagen. Eine Autokratie als konstitutionsloser Staatskörper, in dem sich die einzelnen Organe organismuswidrig verhielten, mußte daher zugrundegehen. In diesem Sinn der Konstitutionslosigkeit hatte Liang auch China als einen „kranken und verkrüppelten Organismus“ bewertet.

„Was ist eine Verfassung? Es handelt sich bei ihr um ein für alle Zeiten unveränderliches Regelwerk, das die Menschen eines Staates, gleich ob Herrscher, Beamte oder Volk, alle gemeinsam bewahren. Es ist die Grundlage aller Gesetzessysteme eines Staates. Gleich welches Gesetz und welche Anordnung einmal geändert werden sollte, sie dürfen nicht von der Grundlage abweichen. Das westliche Wort heißt "Constitution“. Eine Verfassung ist also das Wesen eines Staates. Das konstitutionelle Regierungssystem wird auch als System der eingeschränkten Gewalt bezeichnet. Eingeschränkte Gewalt bedeutet, daß der Herrscher die Gewalt zur Herrschaft hat, so daß sie in diesem Sinn beschränkt ist. Die Beamtenschaft hat die Gewalt der Beamtenschaft, so daß sie in diesem Sinn beschränkt ist. Das Volk hat die Gewalt des Volkes, so daß sie in diesem Sinn beschränkt ist $(\mathrm{x})$."

In diesen Worten Liang Ch'i-ch'aos wird besonders deutlich, daß eine Verfassung in seinem Staatsverständnis nicht eine Deklaration spezifisch demokratischer Staatsformung sein mußte und sollte, sondern gewissermaßen die "anatomische" Beschreibung der Verhaltensgesetze der einzelnen Staatsorgane, aufgrund deren die höchste Koordination und Effizienz des staatlichen Organismus gewährleistet wurde. Die Verfassung verkündete, welcherart Rechte und Pflichten für die Herrschaft und das Volk waren. Wenn alle Glieder diesen Rechtspflichten angemessen nachkamen, dann war das Prinzip der Volkssouveränität vollendet, d. h., der Staat besaß die ihm gemäße Hoheit und Gewalt.

Aus diesem Kausalzusammenhang heraus ist deutlich zu ersehen, warum Liang eine Verfassung für die condito sine qua non seines Staatsmodells hielt. Staatshoheit und Staatsgewalt, die Mittel des modernen Staates zum Konfliktausgleich im Innern und zur geachteten Unabhängigkeit nach außen, konnten nur durch das Volkssouveränitätsprinzip nutzbar gemacht werden, und die Volkssouveränität wiederum war nur durch die Existenz einer Verfassung realisierbar.

„Sobald unsere chinesischen Gelehrten gehört hatten von der Bedeutung einer Beschränkung der Herrschergewalt, da erbleichten sie großenteils. Sie meinen, daß der Herrscher der Höchstgeehrteste eines Landes sei und daß das Volk wie die Beamten alle seine Untertanen seien. Sie wissen nur, daß der Herrscher das Volk in Schranken halten darf. Wie aber hätte jemand gehört, daß das Volk dem Herrscher Schranken auferlegen könnte? Bedeutet denn die Be- 
schränkung des Herrschers durch das Volk nicht nahezu eine Rebellion? Sie wissen nicht, daß der Begriff Beschränkung der Herrschergewalt nicht die Bedeutung hat, daß das Volk sie beschränkt, sondern daß die Verfassung sie beschränkt (y).“

„Warum fürchten sich die Staatsplaner davor, dieses Regierungssystem zu übernehmen? Das ist nicht verwunderlich; denn sie kennen nicht den Unterschied zwischen Volkssouveränität (Min-ch'üan) und Demokratie (Min-chu), und sie behaupten, daß die Volkssouveränität dem von ihr getragenen Herrscher gegenüber feindlich sein muß. So machen sie sich deswegen Sorgen und hassen sie und fürchten sie. Sicherlich zu Recht! Denn sie wissen nicht, daß es eine Verfassungsmonarchie und eine Demokratie gibt. Beide stellen eine Art der Volkssouveränität dar, und der jeweils von ihnen eingeschlagene Weg hat seine besonderen Gründe (z).“

Das enge Interdependenzverhältnis zwischen dem Verfassungsbegriff und dem Prinzip der ganzheitlichen, staatsbezogenen Volkssouveränität mußte notwendigerweise entscheidenden Einfluß auf die Gestaltung des Verfassungswesens ausüben. Da die Grundzüge der Verfassung die besonderen Eigenschaften dieses Souveränitätskonzepts interpretativ darstellen sollten, war die Konstituierung einer parlamentarischen Herrschaftsordnung von vornherein ausgeschlossen. Liang konnte daher nur solche Verfassungsnormen in eine nähere Betrachtung ziehen, die mit seinem chinesischen Staatsmodell harmonisierten und deren Wirklichkeit durch ein verfassungsmonarchisches Staatsdasein gekennzeichnet war. Entsprechende Voraussetzungen boten nur zwei Staaten, nämlich Deutschland und Japan.

„Wenn man sagt, wir sollten Deutschland und Japan zum Vorbild nehmen, so ist das gut. Wenn wir uns alle diese Worte wirklich zu Herzen nähmen, dann wäre das für die Zukunft wirklich ein Glück für China . . . Wie ahmt man Deutschland und Japan also nach? Man sollte ihre Ausdehnung der Volkssouveränität nachahmen, die die Souveränität des Herrschers stützt (aa).“

„Sie fürchten den Begriff Volkssouveränität wie Schlangen. Das ist wirklich beklagenswert. Man kann nicht sagen, daß sie überhaupt nichts um ausländische Dinge wüßten. Sie sind bereits nach Japan gereist und haben japanische Bücher gelesen, und dennoch geben sie solche wirklichkeitsfremden Worte von sich, um die Gesinnung der anderen irrezuführen und den Fortschritt des Landes aufzuhalten ... Ich möchte den Verantwortlichen noch etwas sagen. Wenn diese Herren auch nur einen kleinen Rest an Loyalität und Patriotismus haben, dann sollten sie schleunigst das Wort von der Unterdrückung der Volkssouveränität verwerfen und es nicht mehr im Gedächtnis behalten $(\mathrm{bb}) . “$

„So meinten die Herrscher Preußens und Österreichs zuerst, daß die Errichtung einer Verfassung für sie selbst zum Nachteil wäre. Deshalb wandten sie alle Kraft auf, um sie zu bekämpfen. Nachdem die Verfassung errichtet war, wußten sie erst, daß sie nicht nur keinen Nachteil bringt, sondern auch noch von großem Nutzen ist. Sie bereuten, daß sie vorher selbst Schwierigkeiten bereitet hatten ... Diese Strömung breitete sich nach Osten aus. Japan wurde als erstes erfaßt. Es saugte das Gute auf wie ein Verdurstender. Nachdem seine Grundlagen gefestigt waren, wurde es mächtig. China ist noch wie ein Betrunkener. Es liegt darnieder, und die Zustände sind bis heute elendig (cc).“

Liang Ch'i-ch'ao selbst hatte keinen expliziten Verfassungsentwurf vorbereitet. Ein solches Unternehmen hätte eine juristische und fremdsprachliche Kenntnis 
verlangt, die nur wenige qualifizierte Auslandsfachleute im damaligen China besaßen. Ferner hätte dies seinem eigenen reformerischen Grundverständnis widersprochen, mit dem er immer wieder die Mühseligkeit und Langwierigkeit der planerischen Vorarbeit betont hatte. Dennoch weisen viele Aussagen der Art, wie sie oben zitiert sind, darauf hin, daß er sich eine chineische Nationalstaatsverfassung monarchischen Prinzips erhoffte, die mit der japanischen Meiji-Verfassung vergleichbar war.

"Nun, welche Art von Verfassung China in Zukunft festlegen wird, das kan man heute noch nicht wissen. Welche Art von Verfassung es festlegen sollte, das ist ebenfalls heute noch nicht leicht zu bestimmen. Spricht man über eine Verfassung, dann dürfen die allen Ländern gemeinsamen Grundsätze nicht fehlen wie die Verantwortlichkeit der Minister gegenüber dem Parlament, wie das Wahlrecht des Volkes ... Was diese Punkte angeht, so ist es hinsichtlich der Verantwortlichkeit der Minister gegenüber dem Parlament nicht notwendig, daß es sie einsetzen und absetzen kann, sondern es muß untersuchen und Anklage erheben können, wenn die Untersuchung Unregelmäßigkeiten aufzeigt. Wenn die Regierung dagegen hinterhältig opponiert, dann überprüft es das ebenfalls. Wenn die Regierung eine schlechte Politik betreibt, dann vermag es darauf hinzuweisen. Die Regierung nimmt dann auf seine Aussagen Rücksicht und wagt nicht, rücksichtslos zu sein. Weil sie davor Angst hat, wagt sie nicht, willkürlich zu handeln. So hat das Untersuchen Wirksamkeit (dd).“

„Man muß zuerst entscheiden, welche Art von Regierungssystem unser Staat in Zukunft übernehmen soll. Danach erst können alle Anordnungen und Vorbereitungen demgemäß vorgenommen werden. Wenn man nicht dieserart verfährt, dann gleicht man einem Schiff ohne Kompaß . . . Die Verfassung ist für immer unveränderlich, und sie ist dieGrundlage aller Gesetze. Wenn man sie also einführt, dann muß man auf jeden Fall sorgfältig und genau prüfen und kontrollierten, damit man das beste Ergebnis erzielt. Janpan verwirklichte seine Verfassung im Meiji-Jahr 23 (1890). Es kündigte die Verfassung aber bereits im Meiji-Jahr 13 (1880) an, und ein Rohentwurf wurde bereits im MeijiJahr 5 (1873) erstellt . . . So prüfte man und prüfte nochmals und traf Vorbereitungen und nochmals Vorbereitungen. Wenn China heute so etwas ausführen will, so meine ich, daß es dies in gleicher Reihenfolge vornehmen sollte (ee)."

Bereits zu Beginn dieses Artikels war erwähnt worden, daß die Vorstellungen Linang Ch'i-ch'aos von der Organisation und den geistigen Verhaltensnormen der chinesischen Nation auch unter den Intellektuellen von heute noch eine gewisse Attraktivität ausüben und daß sie ein ständig wiederkehrender Problemgegenstand der politischen Erziehung und der Kulturpolitik in der Volksrepublik sind. Es scheint daher nicht unmöglich, daß diese Vorstellungen bei einem Verebben der ideologischen Energien in der kommunistischen Partei wieder in die öffentliche Diskussion zu rücken vermögen und unter Umständen einen gewissen Einfluß auf die Gestaltung der chineischen Staats- und Gesellschaftssphäre auszustrahlen in der Lage sind.

\section{Anmerkungen zu den Quellentexten:}

Hinsichtlich des Problems der deutschen Originaltexte Bluntschlis und Bornhaks sowie der japanischen Ubersetzungen dieser. Texte und des interpretativen Ver- 
ständnisses Liang Ch'i-ch'aos muß bemerkt werden, daß Liang auf die Ubersetzungen des japanischen Staatswissenschaftlers Katō Hiroyuki beschränkt war.

Gewisse Anzeichen in Liangs Essay "Die Lehren des großen Staatswissenschaftlers Bluntschli" machen es darüber hinaus wahrscheinlich, daß Liang nicht das übersetzte Gesamtwerk selbst studiert hatte, sondern eher auf eine konzentrierte Kurzfassung in Katō Hiroyukis Werk "Neue Theorie des Staatswesens" (Kokutai Shinron) und "Einführung in das verfassungsmäßige Regierungssystem" (Rikken Seitai Ryaku) zurückgegriffen hatte. Diese Vermutung stützt sich vor allem auf die Tatsache, daß die einzelnen Themenbereiche aus dem Gesamtwerk Bluntschlis, die Liang kommentierend anführte, ausschließlich auf ihre nationale Schwerpunktgebung und staatliche Prioritätensetzung hin ausgewählt erscheinen. Eine solche, eher einseitige Akzentuierung des nationalen und staatlichen Moments jedoch spiegelt den ausgewogeneren und maßvolleren Charakter der Bluntschlischen Gesamtlehre nicht vollkornmen wider. Sie deutet $\mathrm{mehr}$ auf eine wissenschaftliche Vorauswertung im Rahmen japanischer Lehrbedürfnisse hin, die dem nationalen Idealkonzept Liangs in noch höherem Maße entsprachen als der deutsche Kontext.

Die große Masse der Schriften Liang Ch'i-ch'aos ist in einem vielbändigen Sammelwerk erfaßt, das den nicht sinnvoll übersetzbaren Titel "Yin-ping-shih he-chi“ trägt und in zwei Serien "Yin-ping-shih wen-chi" und "Yin-ping-shih chuan-chi" unterteilt ist. Diese werden hiernach als "Wen-chi" oder "Chuan-chi" angeführt.

a) Cheng-chih-shüeh ta-chia Po-lun-chih-li chih hsüeh-shuo (Die Lehre des groBen Staatswissenschaftlers Bluntschli), in Wen-chi, Bd. V, Teil 13, S. 85, verfaßt 1903, hiernach Bluntschlis Lehren.

b) Bluntschlis Lehren, S. 81 .

c) Bluntschlis Lehren, S. 83.

d) Ai-kuo lun (Uber den Patriotismus), in Wen-chi, Bd. II, Teil 3, S. 67-68, verfaßt 1899.

e) Über den Patriotismus, S. 75.

f) Hsin-min shuo (Uber die Erneuerung des Volkes), in Chuan-chi, Bd. III, S. 17 , verfaßt 1902 .

g) K'ai-ming chuan-chih lun (Uber den aufgeklärten Absolutismus), in Wenchi, Bd. VI, Teil 17, S. 21, verfaßt 1906, hiernach Absolutismus.

h) Absolutismus, S. 29.

i) Bluntschlis Lehren, S. 87.

j) Bluntschlis Lehren, S. 87-88.

k) Bluntschlis Lehren, S. 88.

l) Bluntschlis Lehren, S. 69-70.

m) Bluntschlis Lehren, S. 71.

n) Bluntschlis Lehren, S. 72.

o) Bluntschlis Lehren, S. 89.

p) Bluntschlis Lehren, S. 81.

q) Absolutismus, S. 35 .

r) Absolutismus, S. 41.

s) Bluntschlis Lehren, S. 87.

t) Absolutismus, S. 64-65.

u) Absolutismus, S. 77-78.

v) Absolutismus, S. 78.

w) Li hsien-fa yi (Anmerkungen zum Entwurf einer Verfassung), in Wen-chi, Bd. II, Teil 5, S. 1, verfaßt 1900, hiernach Verfassung. 
x) Verfassung, S. 1-2

y) Verfassung. S. 2.

z) Verfassung, S. 4.

aa) Ta mou Chün wen Te-kuo Jih-pen ts'ai-yi min-ch'üan shih (Antwort an einen gewissen Herrn, der meint, daß Deutschland und Japan die Volkssouveränität beschränken), in Wen-chi, Bd. IV, Teil 11, S. 55, verfaßt 1902, hiernach Antwort.

bb) Antwort, S. 56-57.

cc) Verfassung. S. 5.

dd) Absolutismus, S. 78.

ee) Verfassung. S. 6. 


\section{BY RÜdiger MachetzKI}

The article discusses the influence of German theories of state on the political thought of Liang Ch'i-ch'ao. Notably scholars like Johann Caspar Bluntschli and Conrad Bornhak made relevant contributions towards a better understanding of the structures and functions of the modern nation-state among the revolutionary Chinese intelligentsia in the early decades of this century. Eminent revolutionary and political theoreticians found access to the traditional German schools of sociopolitical thought through Japanese translations and the scholarly discussion of German models of state and constitution in Japan.

Above all Bluntschli's "organism theory" of the state and its people and the holistic German concept of "people's sovereignty" were of great relevance to Liang's state theorem. Although the heavy influence of liberal, Anglo-Saxon ideals of social and political organization on Liang's political ideas cannot be denied, the article maintains that Liang basically favoured a kind of governmental system which was more adapted to the backward historical state of China's society. The characteristics of this governmental system and its constitutional expression have been put forth by Liang himself particularly in his essays "On Enlightened Absolutism" (K'ai-ming chuan-chih-lun) and "The Theories of the Great German Political Scholar Bluntschli" (Cheng-chih-hsüeh ta-chia Bo-lun-chih-li chih hsüehshuo).

It is in these essays that Liang argues that the theories of the constitutional state as presented by Bluntschli and Bornhak constitute the most suitable model for a modernized Chinese state and people.

\section{China and International Law: 21 Years of Treaty Practice}

\section{By Oscar Weggel}

Are there one, two (socialist - bourgeois) or three (socialist - bourgeois - general) systems of international law according to Chinese views? All these possibilities were discussed and acknowledged during the fifties. The answer, however, depends on practice. The socialist - non-socialist states treaty ratio of the People's Republic of China in 1964/65 was 57:126, and from 1966 through 1971 even 72: 170. Thus China's treaty practice draws a sharp line between socialist and non-socialist countries. But does China neglect the "pacta sunt servanda" - principle vis-à-vis non-socialist states? That depends on the character of the treaty. As a general rule, purely commercial treaties as well as development-aid-commitments are being faithfully fulfilled though there are five exceptions to the latter category, i. e. Ghana, Indonesia, Cambodia under Lon Nol, Burma, and the Mongolian People's Republic. The most problematic treaties, however, are the purely political ones, such as the 17 friendship treaties concluded up to now. Particularly in the cases of India, Indonesia, Burma and Cambodia China did not feel impelled to meet her treaty obligations. Regarding these purely political treaties China is sometimes inclined to advocate the existence of two different systems of international law. As to the rest, however, her practice of treaties is a very clear manifestation of her acknowledging a coherent international law system. 